\title{
A Novel "Petaloid" Mutant of Torenia (Torenia fournieri Lind. ex Fourn.) Bears Double Flowers through Insertion of the DNA Transposon Ttf1 into a C-class Floral Homeotic Gene
}

\author{
Takaaki Nishijima ${ }^{1,2 *}$, Tomoya Niki ${ }^{1,2}$ and Tomoko Niki ${ }^{1}$ \\ ${ }^{1}$ NARO Institute of Floricultural Science, Tsukuba 305-8519, Japan \\ ${ }^{2}$ Graduate School of Life and Environmental Sciences, University of Tsukuba, Tsukuba 305-8577, Japan
}

\begin{abstract}
A double-flowered torenia (Torenia fournieri Lind. ex Fourn.) mutant, "Petaloid", was obtained from selfed progeny of the "Flecked" mutant, in which the transposition of the DNA transposon Ttf1 is active. A normal torenia flower has a synsepalous calyx consisting of 5 sepals, a synpetalous corolla consisting of 5 petals, 4 distinct stamens, and a syncarpous pistil consisting of 2 carpels. In contrast, a flower of the "Petaloid" mutant has 4 distinct petals converted from stamens, whereas the calyx, corolla, and pistil remain unchanged. The double-flower trait of the "Petaloid" mutant was unstable; some or all of the 4 petals converted from stamens frequently reverted to stamens. Furthermore, most $S_{1}$ plants obtained from self-pollination of the somatic revertant flower bore only normal single flowers. In petals converted from stamens, expression of the C-class floral homeotic gene T. fournieri FARINELLI (TfFAR) was almost completely inhibited. This inhibition was caused by insertion of $T t f 1$ into the 2 nd intron of $T f F A R$, whereas reversion of converted petals to stamens was caused by excision of $T t f 1$ from TfFAR. The clear correspondence of the TfFAR genotype to the floral phenotype suggested that homozygous $T f F A R$ alleles with the $T t f 1$ insertion caused the mutant phenotype. In contrast, TfFAR was moderately expressed in the pistil of the "Petaloid" mutant, leaving the pistil unchanged. We succeeded in inactivating $T t f 1$ transposition by cross-pollination between mutant and normal-type plants to genetically separate the transposon $T t f 1$ from the unidentified factor activating its transposition, which made the "Petaloid" mutation more stable.
\end{abstract}

Key Words: En/Spm superfamily, euAG lineage gene, mutable allele, TfFAR.

\section{Introduction}

Torenia (Torenia fournieri Lind. ex Fourn.) is primarily used as a bedding or potted plant in the summer in Japan. Torenia plants branch vigorously, and bear many flowers, forming a neat, compact arrangement (Morise, 2001; National Agriculture and Food Research Organization, 2006). Torenia grows well under a wide range of light intensities, from direct sunlight to weak light in the shade, and is also highly tolerant of high temperatures during mid-summer, which have been increasing by recent climate change. Breeding for flower colour variation has produced torenia cultivars with

Received; September 1, 2015. Accepted; November 9, 2015. First Published Online in J-STAGE on July 23, 2016.

This work was supported by JSPS KAKENHI Grant Number 25292026 .

* Corresponding author (E-mail: takaaki@affrc.go.jp). white, pink, red, and yellow flowers, in addition to the original violet flower of the wild T. fournieri. Several breeding techniques have been employed to further extend the genetic variation of torenia. Interspecific crosses among Torenia species have increased the variety of flower colours (Miyazaki et al., 2007). However, fertile hybrids from interspecific crosses are limited, and variation in floral morphology is relatively poor among Torenia species. Although heavy ion-beam radiation and genetic transformation had increased the available variation in flower colour and morphology of T. fournieri (Aida et al., 2000; Sasaki et al., 2008), variation in floral morphology remains limited. Recently co-overexpression of B-class floral homeotic genes TfDEF (TfDEFICIENS) and TfGLO (TfGLOBOSA) induced conversion of sepals into petaloid organs, resulting in a kind of double flower (Sasaki et al., 2014). However, commercial use of transgenic torenia would be difficult at least in Japan because of the substantial 
cost for official registration and potential negative public perception (Oshima et al., 2010). Thus, there have been no double-flowered or large flowered torenia cultivars. If this limitation could be overcome, and a highly attractive appearance could be combined with the excellent growth properties mentioned above, the commercial value of torenia would increase significantly.

Although double flowers can result from several different morphological changes, the most frequently observed is the conversion of stamens and carpels into petals (Nishijima, 2012). This conversion is induced by a reduction in C-class gene function in floral homeotic genes. Floral organ identity is determined based on the mechanism described by the ABC model (Bowman et al., 1991; Coen and Meyerowitz, 1991). Combined A- and B-class gene expression establishes petal identity in whorl 2, and B- and C-class gene expression establishes stamen identity in whorl 3. Carpel identity is established by $\mathrm{C}$-class gene expression alone in whorl 4. A- and C-class genes antagonistically control the expression of each other (Drews et al., 1991; GustafsonBrown et al., 1994), and avoid overlap of A- and Cclass gene expression. Thus, a reduction in C-class gene function permits A-class gene function in whorl 3, resulting in the conversion of stamens into petals. Further reduction in C-class gene function induces the indeterminate development of flowers in whorl 4 because reduction in C-class gene function promotes WUS expression in the floral meristem, preserving the meristematic competence of the floral bud, and inducing indeterminate development (Lenhard et al., 2001; Lohmann et al., 2001). These mechanisms were elucidated mainly in Arabidopsis thaliana and Antirrhinum majus. With regard to the reduction or loss of C-class gene function in A.majus, which belongs to Lamiales as torenia, a loss-of-function mutant of the C-class gene PLENA (PLE) causes the conversion of stamens into petals in A. majus (Bradley et al., 1993). Furthermore, double mutation of ple and another C-class gene, farinelli (far) decreases determinacy of flower development, inducing double flowers with many petaloid organs.

Spontaneous mutations caused by transposable elements have been important mutagens in breeding floricultural plants (Inagaki et al., 1994; Matsubara et al., 2005; Nakajima et al., 2005; Nitasaka, 2003, 2007). However, transposable elements with high transposition activity often produce unstable traits (e.g. Goodrich et al., 1992; Inagaki et al., 1994). If transposition activity is moderate, and does not disrupt the practical uniformity of cultivars, transposable elements function as a useful mutagen for breeding (Matsubara et al., 2005; Nakajima et al., 2005; Nitasaka, 2003, 2007). We previously isolated a torenia mutant in which transposition of an Enhancer/Supressor-Mutator (En/Spm)-like transposon, Ttf1 (Transposon T.fournieri 1), is activated (Nishijima et al., 2013). In this mutant, named
"Flecked", Ttfl is inserted in the 2nd intron of TfMYB1, which encodes a R2R3-MYB transcription factor promoting anthocyanin biosynthesis in petals. The insertion of Ttfl reduces TfMYB1 expression, which fades petal colour from violet to pale violet. Spontaneous excision of Ttf1 from TfMYB1, i.e. somatic reversion, causes small violet spots, semicircular violet sectors, or solid violet petal limbs. Self-pollination of complete somatic revertant flowers with solid violet petal limbs results in a high proportion of germinal revertant $S_{1}$ plants. In the $S_{1}$ plants, the allele in which Ttfl is newly integrated may become homozygous, and the plants may express the new mutant phenotype.

We isolated, from the selfed progeny of "Flecked" mutant, a double-flowered mutant in which stamens were converted to petals. This is the first report of a double-flowered torenia mutation. This mutant, named "Petaloid", is unstable, like the mutant "Flecked". In this paper, we report the molecular mechanism underlying the double-flower trait of this mutant. Furthermore, we propose a method to stabilize the mutant trait in order to use the Ttfl-induced mutants as breeding material.

\section{Materials and Methods}

\section{Plant material and isolation of the "Petaloid" mutant}

For isolation of the "Petaloid" mutant of torenia (T. fournieri Lind. ex Fourn.), flowers with apparent somatic reversion, i.e. flowers of the "Flecked" mutant with a solid violet lower lip (Nishijima et al., 2013) were self-pollinated by hand. We pollinated 2 flowers per plant and approximately 280 plants in total. Approximately $2000 \mathrm{~S}_{1}$ plants were screened for novel mutations. The growth conditions of the plants were as follows. The seeds were planted in 288-cell plug trays (54 cm in length and $28 \mathrm{~cm}$ in width; each cell $1.8 \mathrm{~cm}$ in length and width and $3 \mathrm{~cm}$ in height) filled with a horticultural medium (Primemix; Sakata Seed Co. Ltd., Tokyo, Japan). To isolate novel mutants, seedlings were transplanted into 72 -cell plug trays $(54 \mathrm{~cm}$ in length and $28 \mathrm{~cm}$ wide; each cell $4 \mathrm{~cm}$ long, $4 \mathrm{~cm}$ wide and $6 \mathrm{~cm}$ high) filled with Kureha-Engei-Baido (Kureha Chemical Industry Co. Ltd., Tokyo, Japan) and Primemix [1:1 $(\mathrm{v} / \mathrm{v})]$. Seedlings for genetic and molecular analysis and stabilization of the double-flowered trait, as described below, were planted in pots $(9 \mathrm{~cm}$ in diameter and $12 \mathrm{~cm}$ deep; 3 seedlings per pot) filled with the same medium as that used in the 72-cell plug trays to obtain highly stable and uniform growth. The plants were grown in a glasshouse with natural sunlight. The temperature was kept between $18^{\circ} \mathrm{C}$ and $32^{\circ} \mathrm{C}$. A slowreleasing coated fertilizer (Ecolong 100; Chisso Co., Tokyo, Japan) was used for top-dressing.

\section{Microscopic observation}

Young flower buds were fixed in a mixture of $70 \%$ $(\mathrm{v} / \mathrm{v})$ ethanol and $5 \%(\mathrm{v} / \mathrm{v})$ acetic acid at $4{ }^{\circ} \mathrm{C}$ overnight, 
and then dehydrated through a graded ethanol series and transferred to $t$-butanol/ethanol $(1: 1 \mathrm{v} / \mathrm{v})$. The samples were then transferred to $100 \% t$-butanol, freezedried, and subjected to scanning electron microscopy (SEM) (VE-7800; Keyence, Osaka, Japan). Floral stages were identified according to Nishijima and Shima (2006). Surface structures of mature floral organs were observed by directly subjecting the raw samples to SEM.

\section{Genetic analysis}

A single normal type (NT) flower of "Flecked", mutant-type (MT), and apparent somatic revertant (SR) flowers of "Petaloid" were cross-pollinated by hand in the combinations shown in Table 1. Six flowers from each of the seed and pollen parent plants were used for crossing.

\section{Expression analysis of floral homeotic genes}

To investigate expression of floral homeotic genes in NT, MT, and SR flowers, the corolla, stamen, and pistil were collected from flower buds during the early and late portions of the petal development stage (stage 6; Nishijima and Shima, 2006). Corolla length was 4-6 and $14-16 \mathrm{~mm}$ early and late in stage 6 , respectively. The samples were frozen in liquid nitrogen, and stored at $-80^{\circ} \mathrm{C}$ until use. Total RNA was obtained using an RNeasy Plant Mini Kit (QIAGEN K. K., Tokyo, Japan). cDNA was synthesized using a PrimeScript 1 st strand cDNA Synthesis Kit (TakaraBio, Shiga, Japan). For total RNA extraction for cDNA synthesis, we treated the samples with DNase to avoid potential contamination with genomic DNA. Gene-specific primers for torenia floral homeotic genes and the actin gene [T. fournieri ACTIN3 (TfACT3); AB330989], which was used as an internal control, were designed for the 3'terminal regions of the open reading frame and the 3'untranslated regions of each gene. Primer sequences and the lengths of PCR products used for the quantitative real-time PCR reactions were as follows: forward 5'-GCTTTGCTGCATGATGATATA-3' and reverse 5'GCGTTGTTTTGTTGCATCT-3' for TfSQUA (TfSQUAMOSA, $151 \mathrm{bp} ; \mathrm{AB} 359949)$, forward 5'GGTACTACTAATTTCGTAGGG-3' and reverse 5'-
TAATATGGATCGAAATCATC-3' for TfDEF (103 bp; AB359951), forward 5'-CGAATCTTCAGGAACGTT TC-3' and reverse 5'-AAGGTTTTGGCTTAACGAG AG-3' for TfGLO (111 bp; AB359952), forward 5'CCTTTGGCTGTTAGGATG-3' and reverse 5'-GACA CAGCCCGAGTCGATGAG-3' for TfPLE1 (172 bp; 359954), forward 5'-ATGGGATCCTCTGCTGATTAT$3^{\prime}$ and reverse 5'-TTCAAATTGAACAACACATGG-3' for TfFAR (129 bp; AB359953) and forward 5'TGCAGTAAAGTGTATTGTGGAAG-3' and reverse 5'-GGAACTATCTGGGTAGGATC-3' for TfACT3 (145 bp). We previously isolated two other class-C floral homeotic genes, TfPLE2A (AB359955) and TfPLE2B (AB359956); however, we did not analyze these genes because their deduced amino acid sequences have marked truncation. Expression of the genes was quantified by a quantitative real-time PCR (qPCR) (Thermal Cycler Dice TP-800; TakaraBio) and SYBR Premix Ex Taq (TakaraBio). The reactions were cycled with a preincubation step of $10 \mathrm{~s}$ at $95^{\circ} \mathrm{C}$, followed by 45 cycles of $5 \mathrm{~s}$ at $95^{\circ} \mathrm{C}$ and $30 \mathrm{~s}$ at $60^{\circ} \mathrm{C}$. The raw data were analysed with Thermal Cycler Dice Real Time System Software (TakaraBio). Plasmids (pGEMT Easy vector; Promega, Madison, WI, USA) harbouring cDNAs of the target genes were used to obtain standard curves. The ratio of the expression of each gene to that of TfACT3 was calculated. The expression analysis was independently conducted in triplicate.

Molecular cloning of the gene responsible for the "Petaloid" mutation

Stamens from NT flowers, stamens converted to petals from MT flowers and stamens from SR flowers were collected late in stage 6 . The samples were frozen in liquid nitrogen and stored at $-80^{\circ} \mathrm{C}$ until use. Genomic DNA was extracted using a MagExtractor Plant Genome Kit (TOYOBO, Osaka, Japan). The genomic sequence, including the full-length open reading frames of class-C floral homeotic genes, was amplified by nested PCR using the following primer sets; TfPLE1, 1st PCR: forward 5'-CTGCAACTCTCCTGTCCACAA-3' and reverse 5'-GAACAAAAGCCATGCAATGA-3', 2nd PCR: forward 5'-AAATCTCTTTCTTTCTCGCT CTC-3' and reverse 5'-CACTTAATAACGTGTGCG

Table 1. Trait segregation of $F_{1}$ and $S_{1}$ plants derived from crosses among normal type flowers (NT), mutant type flowers (MT), and flowers apparently with complete somatic reversion (SR)

\begin{tabular}{ccc}
\hline \hline Cross & $\begin{array}{c}\text { Plant solely with the NT flower } \\
\text { (No. of plants (\%)) }\end{array}$ & $\begin{array}{c}\text { Plant with MT flower } \\
(\text { No. of plants }(\%))\end{array}$ \\
\hline $\mathrm{MT} \times \mathrm{NT}$ & $48(100)$ & $0(0)$ \\
$\mathrm{NT} \times \mathrm{SR}$ & $51(100)$ & $0(0)$ \\
$\mathrm{SR} \times \mathrm{NT}$ & $47(100)$ & $0(0)$ \\
$\mathrm{MT} \times \mathrm{SR}$ & $31(63)$ & $18(37)$ \\
$\mathrm{SR} \times \mathrm{SR}$ & $43(84)$ & $8(16)$ \\
\hline
\end{tabular}

${ }^{\text {z }}$ Plants with MT flowers include ones bearing both MT and SR flowers within a plant. 
ACAC-3'; TfFAR, 1st PCR: forward 5'CTGCAACTCTCCTGTCCACAA-3' and reverse 5'GTAAATAATTGTCCCTTGACTTC-3', 2nd PCR: forward 5'-TCAGAAAAGCAGAAACTTGAGC-3' and reverse 5'-AATATAGCAGCAATCGAAGGTT-3'. The PCR fragments were cloned into pGEM T-easy vectors (Promega), and analysed using a DNA sequencer (ABI 3100 Genetic Analyser; Life Technologies Co., Foster City, CA, USA).

Analysis of TfFAR genotype correspondence to the mutant phenotype

$\mathrm{S}_{1}$ plants descended from somatic revertant flowers were used. We analysed $12 \mathrm{~S}_{1}$ mutant plants bearing both MT and apparent SR flowers on a single plant and 12 apparent germinal revertant (GR) plants bearing only revertant flowers. Petals converted from stamens were collected from MT flowers of mutant plants, whereas stamens were collected from apparent GR plants. Genomic DNA was extracted as described above. qPCR analysis was performed as described above to confirm the TfFAR genotype. In the following text, the allele of TfFARl bearing the Ttfl insertion is indicated as $f^{I t f l}$, whereas the allele of TfFAR without Ttfl insertion is indicated as $F$. We used the following 2 primer sets. Primer set A, forward 5'-ATTTTTCTGTC GCCGGATCC-3' and reverse 5'-CTGATACATCTGAA ACTCAGATCTGAAG-3', amplifies the 127-bp sequence between the right subterminal region of Ttfl and the 2nd intron of TfFAR in $f^{T t f l}$, but does not amplify any sequence of $F$. Primer set B, forward 5'-GTAAAA CATTAGTAAAGGGCAAAGTGTC-3' and reverse 5'CTGATACATCTGAAACTCAGATCTGAAG-3' (identical to the reverse primer of set A), amplifies a 149-bp sequence in $F$, but does not amplify any sequence in $f^{I t f l}$ because the sequence to be amplified is too long (3042 bp). Quantitative real-time PCR analysis was performed as described above using genomic DNA as a template. Plasmids (pGEM-T Easy vector) harbouring genomic sequences of $f^{\text {Ttf } f}$ and $F$ were used to obtain standard curves. TfACT3 was employed as an internal standard using the primers described above. The genomic sequence amplified by this primer set was the same length as the cDNA sequence.

\section{Inactivation of a Ttfl excision from TfFAR}

Pollen from "Common Violet" in which Ttf1 transposition is inactive was placed on the stigmas of MT flowers of "Petaloid" by hand. The resultant $4 F_{1}$ plants were self-pollinated to produce $F_{2}$ plants. Stability of the double-flower trait was evaluated in $\mathrm{F}_{2}$ plants. The TfFAR genotype of $\mathrm{F}_{2}$ plants was analysed by qPCR as described above. Petals converted from stamens from 3 mutant flowers per plant were analysed.

\section{Results and Discussion}

\section{Isolation of the "Petaloid" mutant}

We obtained a double-flowered mutant from among approximately $2000 \mathrm{~S}_{1}$ plants of the "Flecked" mutant. Since this double-flowered mutant occasionally bore revertant flowers with normal stamens, we placed pollen from revertant flowers on the stigmas of mutant flowers to obtain $\mathrm{M}_{2}$ seeds. A similar method was repeated in the following generations to maintain the mutant. Double-flowered $\mathrm{M}_{4}$ plants were selected and used in the experiments.

\section{Morphological characterization of the "Petaloid" mu- tant}

A normal-type (Fig. 1, NT) torenia flower has 4 stamens, and the basal part of the filaments are fused to the corolla tube. The upper two stamens are located on the margin of the upper and lower lips, whereas the lower two are located on the lower lip (indicted by a black arrowhead with white margin in Figure 1). In contrast, the stamens of the double-flowered mutant (Fig. 1, MT, i.e. mutant type) were converted to petals (indicated by a white arrowhead with black margin in Figure 1). In NT flowers, the epidermal cells of the anther and filament were flat (Fig. 2E, F). In contrast, both the adaxial and abaxial epidermal cells were conelike in petals converted from stamens (Fig. 2C, D), identical to other petal cells (Fig. 2A, B). These results suggest that stamens of the MT flower are completely converted to petals. The double-flower trait of the mutant was unstable; all 53 plants tested bore SR flowers until 42 days after the first flower opened. Once an SR flower developed, further apical flowers, seemingly generated from the same cell lineage, were mostly SR. Flowers with both stamens and petals converted from stamens (Fig. 1, IR, i.e. incomplete somatic revertants) occurred frequently, perhaps caused by localized reversion of the double-flowered trait. Because this mutant trait resembles that of the "Petaloid" of Japanese morning glory (Ipomoea nil), which is caused by mutation of a C-class floral homeotic gene (Nitasaka, 2007), we named the torenia mutant and its double-flowered trait "Petaloid", although the mutant trait of the "Petaloid" of Japanese morning glory is stable.

In developing flower buds, no morphological differences were observed between NT and MT plants before differentiation of the corolla, stamen, and pistil primordia (Fig. 3, in stage 2, the sepal initiation stage); however, in stage 4, the sex organ and corolla initiation stage, stamen primordia grew thicker than petal primordia in NT flower buds, whereas there were no clear morphological differences between petals converted from stamens and petals in MT flower buds. In stage 5, the early corolla development stage, globular anthers and plate-like petals were clearly distinguishable in NT flower buds; however, both petals converted from sta- 


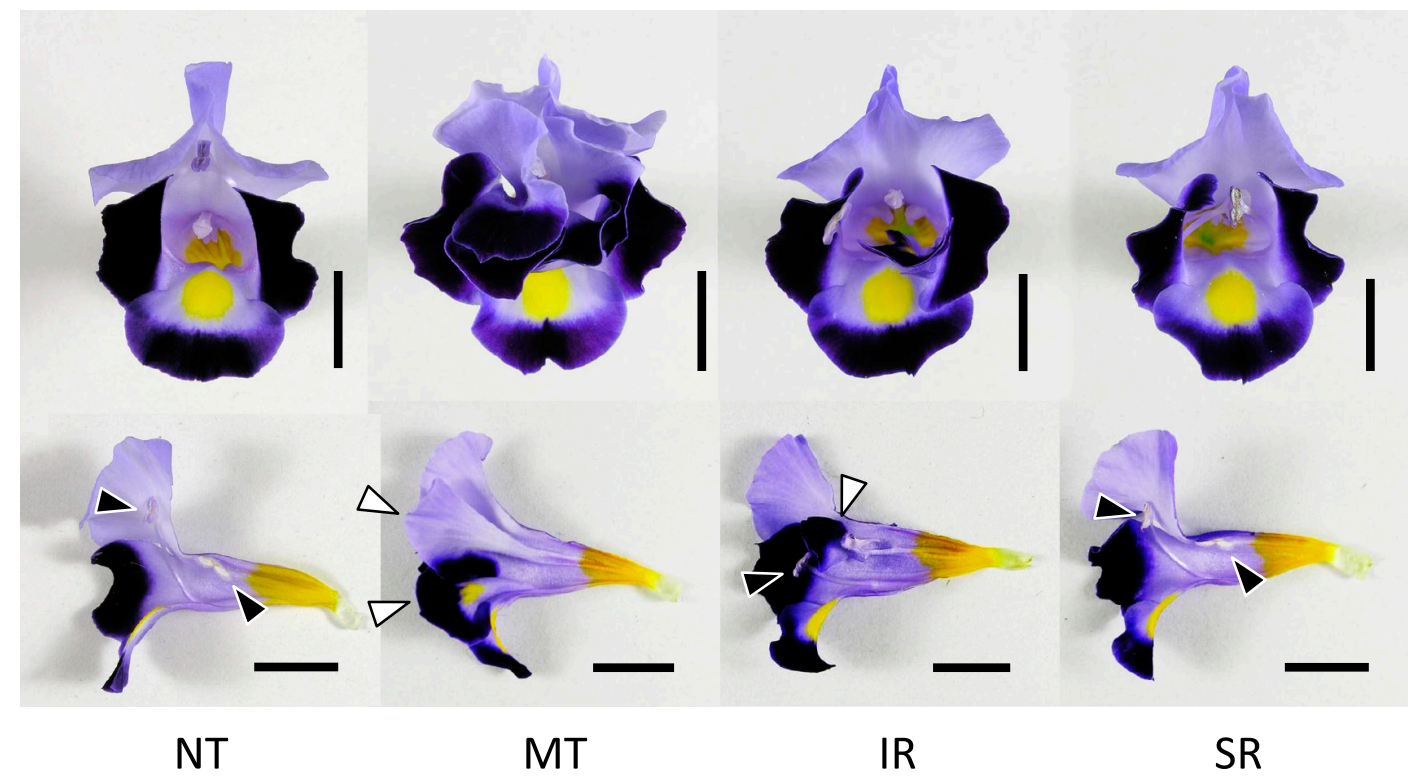

Fig. 1. Flower of the "Petaloid" torenia mutant. Upper column shows a front view of the flowers, and the lower column shows a side view of longitudinally bisected flowers. NT, normal type; MT, mutant type; IR, flower with incomplete somatic reversion; and SR, flower with complete somatic reversion. Black arrowhead with white margin indicates stamen, while white arrowhead with black margin indicates the petal converted from stamen. Black bar indicates $10 \mathrm{~mm}$.
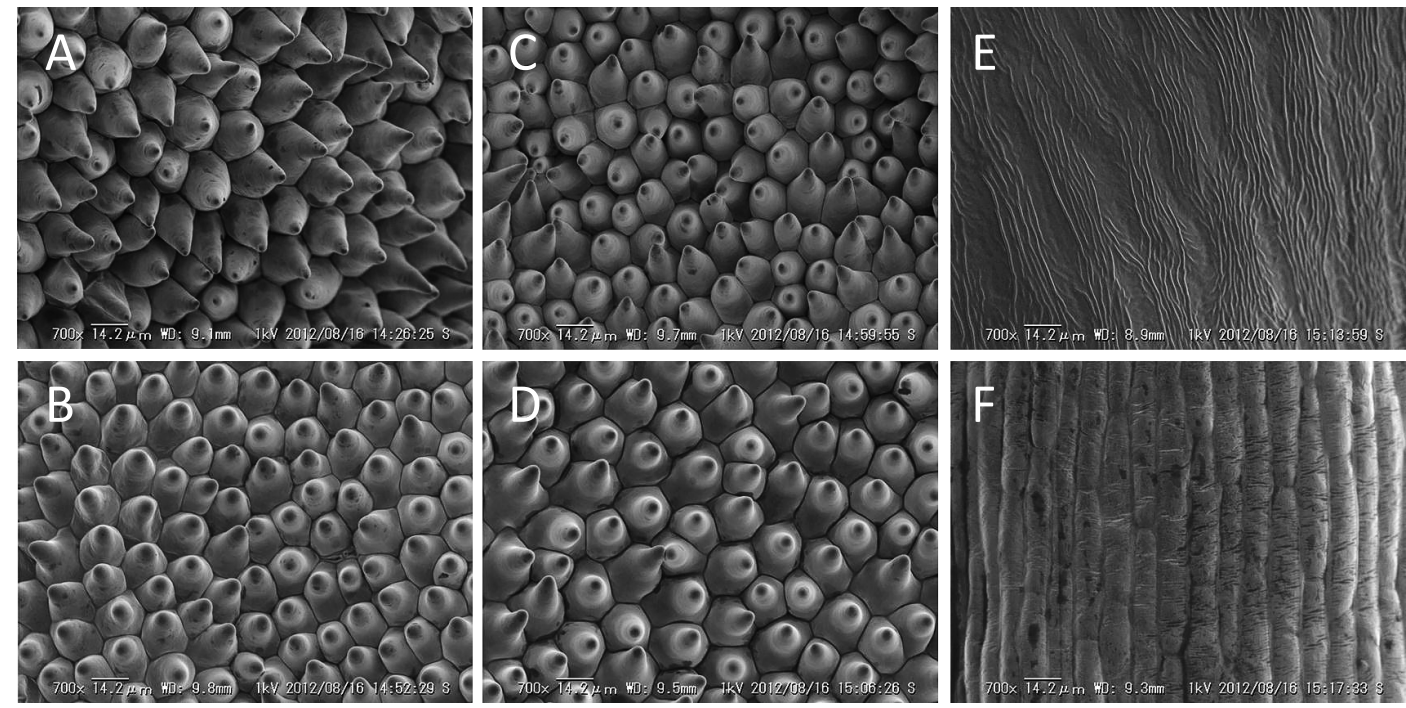

Fig. 2. Scanning electron microscopy of floral organs of the "Petaloid" mutant. Adaxial (A) and abaxial (B) petal faces of the normal-type flower; adaxial (C) and abaxial (D) faces of a petal converted from the stamen in the mutant-type flower; anther (E) and filament (F) of the normal-type flower.

mens and petals were plate-like in MT flower buds, although petals converted from stamens were narrower than petals. In stage 6 , the middle corolla development stage, the pollen sac was clearly observable in stamens of NT flower buds, whereas petals developed a wide plate-like shape. In contrast, both petals converted from stamens and petals of MT flower buds developed the plate-like shape characteristic of petals. No differences in pistil development were observed between NT and MT flowers.

\section{Genetic analysis of the double-flowered trait}

All $\mathrm{F}_{1}$ plants derived from the cross between the MT and NT flowers bore only NT flowers (Table 1). When an NT flower was crossed with an SR flower, all $F_{1}$ plants also bore only NT flowers. These results suggest that the double-flowered trait is recessive. In contrast, the cross between MT and SR flowers and selfpollination of an SR flower resulted in $\mathrm{F}_{1}$ and $\mathrm{S}_{1}$ plants bearing MT flowers (i.e. MT plants). In these combinations, the resulting percentage of MT plants was higher in the cross between MT and SR flowers than in the 


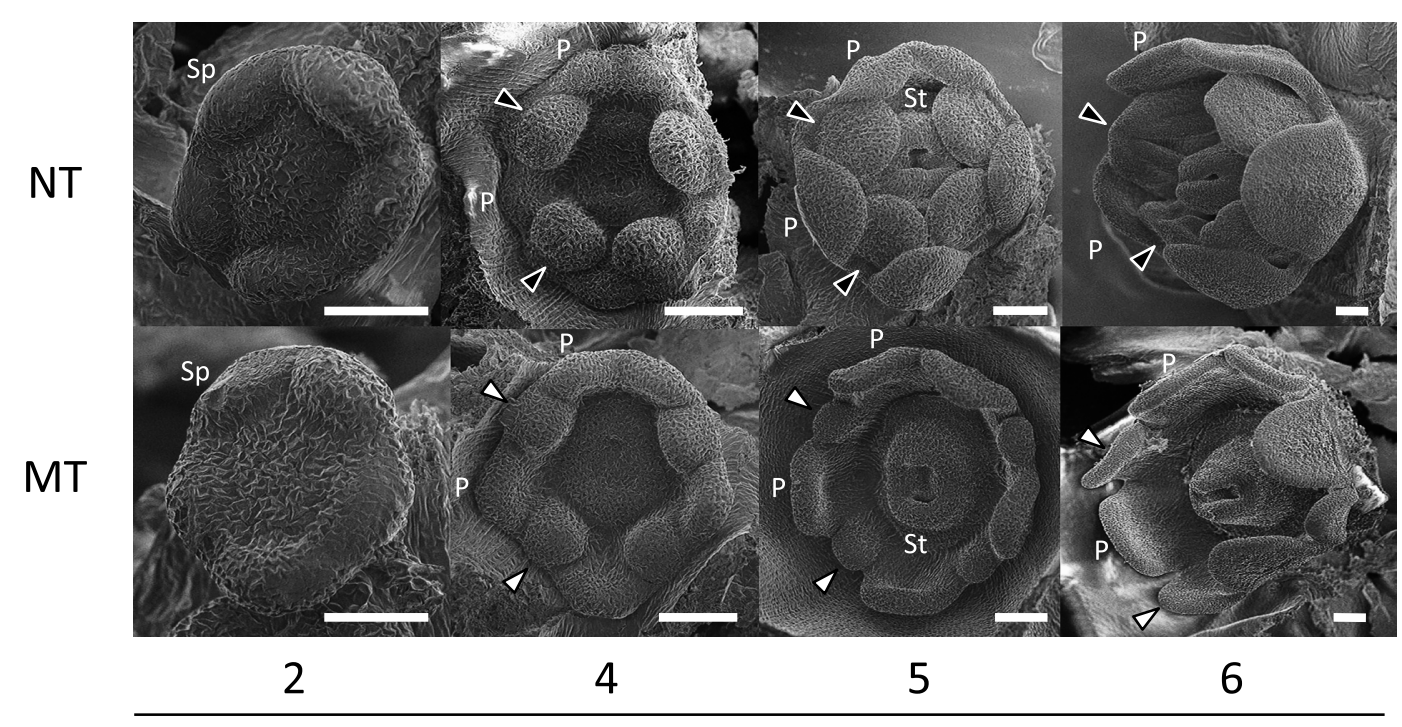

Floral stage

Fig. 3. Floral development of the "Petaloid" mutant. NT, normal type; and MT, mutant type. Black arrowhead with white margin indicates the stamen, while white arrowhead with black margin indicates a petal converted from the stamen. P, petal; St, stigma; and Sp, sepal. White bar indicates $50 \mu \mathrm{m}$.

self-pollinated SR flowers. If the double-flowered mutation is caused by a single recessive allele $d, 50 \%$ of the $\mathrm{F}_{1}$ plants derived from a cross between the MT $(d d)$ and SR $(D d)$ flowers would be MT $(d d)$ plants; however, the actual ratio of the MT $F_{1}$ plants was $37 \%$. Similarly, $25 \%$ of the $S_{1}$ plants derived from self-pollination of the SR $(D d)$ flowers would be MT $(d d)$ plants; however, the actual ratio of MT $S_{1}$ plants was $16 \%$. The lower observed percentages of MT plants compared with the theoretical estimations indicates substantial reversion of the mutant allele.

\section{Expression of floral homeotic genes in floral organs of the "Petaloid" mutant}

In NT flower buds in early stage 6, petals had marked expression of the B-class floral homeotic genes $T f D E F$ and $T f G L O$, as predicted by the ABC model; however, expression of the A-class floral homeotic gene TfSQUA was slight. Stamens showed marked expression of TfDEF and TfGLO and of the C-class floral homeotic genes TfPLE1 and TfFAR (Fig. 4), whereas TfPLE1 and TfFAR were the main floral homeotic genes expressed in pistils. These results are as predicted by the ABC model, except expression of TfSQUA was slight in petals. The expression profile of these genes in stamens and pistils were essentially the same in late stage 6 (Fig. 5). At this stage, the tube and limb of petals were separately analysed. Marked expression of $T f D E F$ and TfGLO was observed both in the tube and limb, but only slight or no TfSQUA expression was detected as in petals in the early stage 6 (Fig. 4). Although TfSQUA is markedly expressed in petal primordia (Niki et al., 2012), our results show that TfSQUA is barely ex- pressed in more developed petals.

The basal portion of the stamen is fused to the corolla tube in late stage 6 , meaning each tube sample contains a small amount of filament; however, the expression of TfPLE1 and TfFAR, which are expressed in stamens, was very low in the corolla tube. This is possibly because expression of C-class genes is relatively low in filaments, as shown in A. majus (Yamaguchi et al., 2010).

In petals converted from stamens in MT flower buds, expression of TfPLE1 and TfFAR almost completely disappeared in both early and late stage 6 , whereas $T f D E F$ and TfGLO were markedly expressed. Furthermore, TfSQUA was significantly expressed in early stage 6 . Therefore, the expression profile of floral homeotic genes follows the "petal" pattern in early stage 6 ; it is clear that this gene expression profile converted stamens into petals.

In the pistils of MT flower buds, mainly TfPLE 1 and $T f F A R$ were expressed, both in early and late stage 6; however, TfFAR expression was lower than in NT flower buds (Figs. 4 and 5). In contrast, the expression of TfPLE1 was not lower than in NT flower buds. The expression profile of petals of MT flower buds was almost identical to that of NT flower buds, with the exception that in early stage 6, TfSQUA was significantly expressed (Fig. 4). The "Petaloid" mutation further decreased the slight TfFAR expression in the petals, although expression was too slight to clearly show the decrease in Figure 4. This decrease may have released repression of TfSQUA expression (Fig. 4) because expression of A- and C-class genes is reciprocally antagonistic (Drews et al., 1991; Gustafson-Brown et al., 1994). 


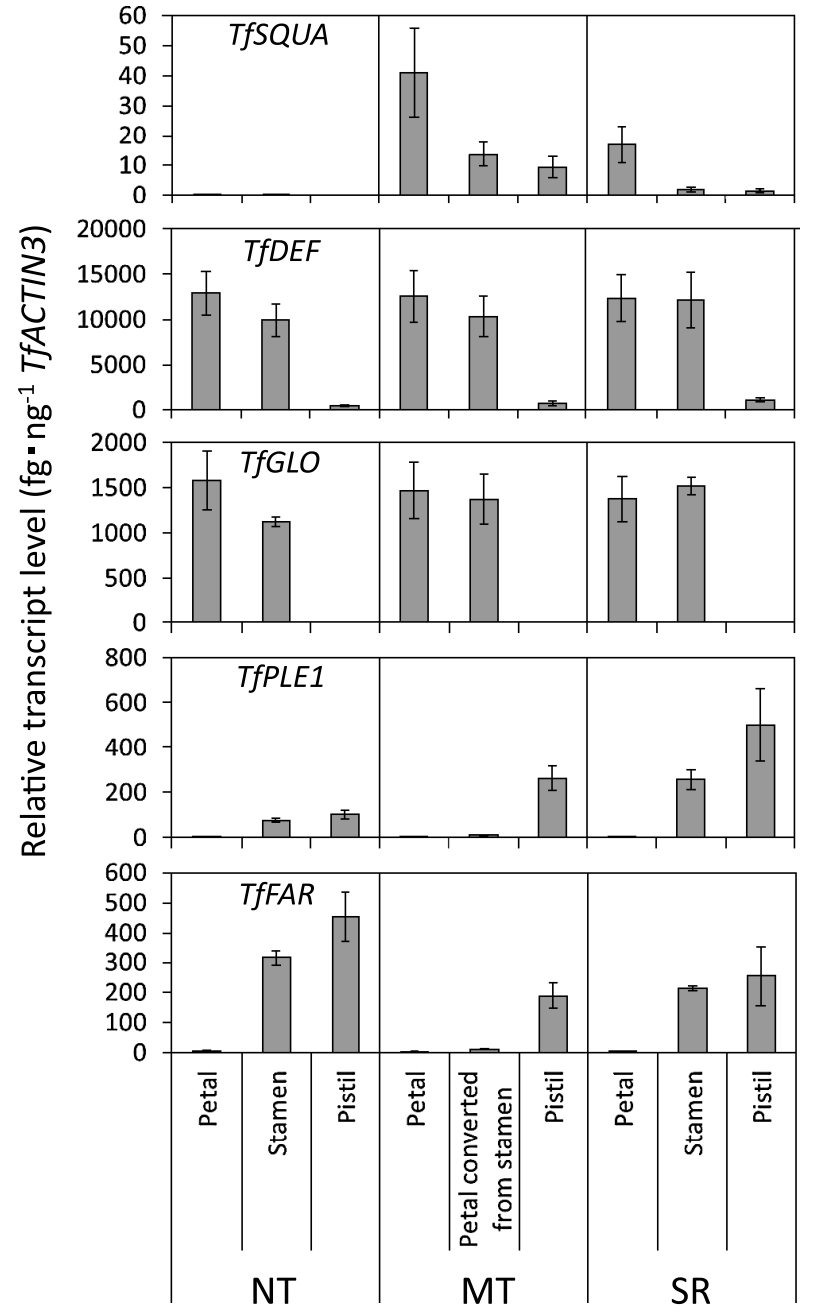

Fig. 4. Expression of floral homeotic genes in young flower buds of the "Petaloid" mutant. Flower buds in the early petal development stage (early stage $6,4-6 \mathrm{~mm}$ corolla length) were used for the experiment. NT, normal type; MT, mutant type; and SR, flower with complete somatic reversion. Vertical bars indicate SE $(n=3)$.

The expression profile of floral homeotic genes in SR flower buds was essentially identical to that of NT flower buds in both early and late stage 6 (Figs. 4 and 5); however, TfSQUA expression was higher in petals than in NT flower buds in early stage 6 , likely due to lower levels of TfFAR expression in SR petals than in NT petals, although expression was too slight to clearly show the difference in Figure 4. It should be noted that TfPLE1 expression was higher in stamens and pistils in SR flower buds than in NT flower buds.

Because stamens of "Petaloid" were converted to petals, it was expected that a C-class floral homeotic gene had mutated, as observed in the aforementioned "Petaloid" morning glory (Nitasaka, 2007). As expected, TfPLE1 and TfFAR expression in MT petals converted from stamens were almost completely absent. In contrast, substantial expression of these genes remained in pistils, which may have inhibited the conver-

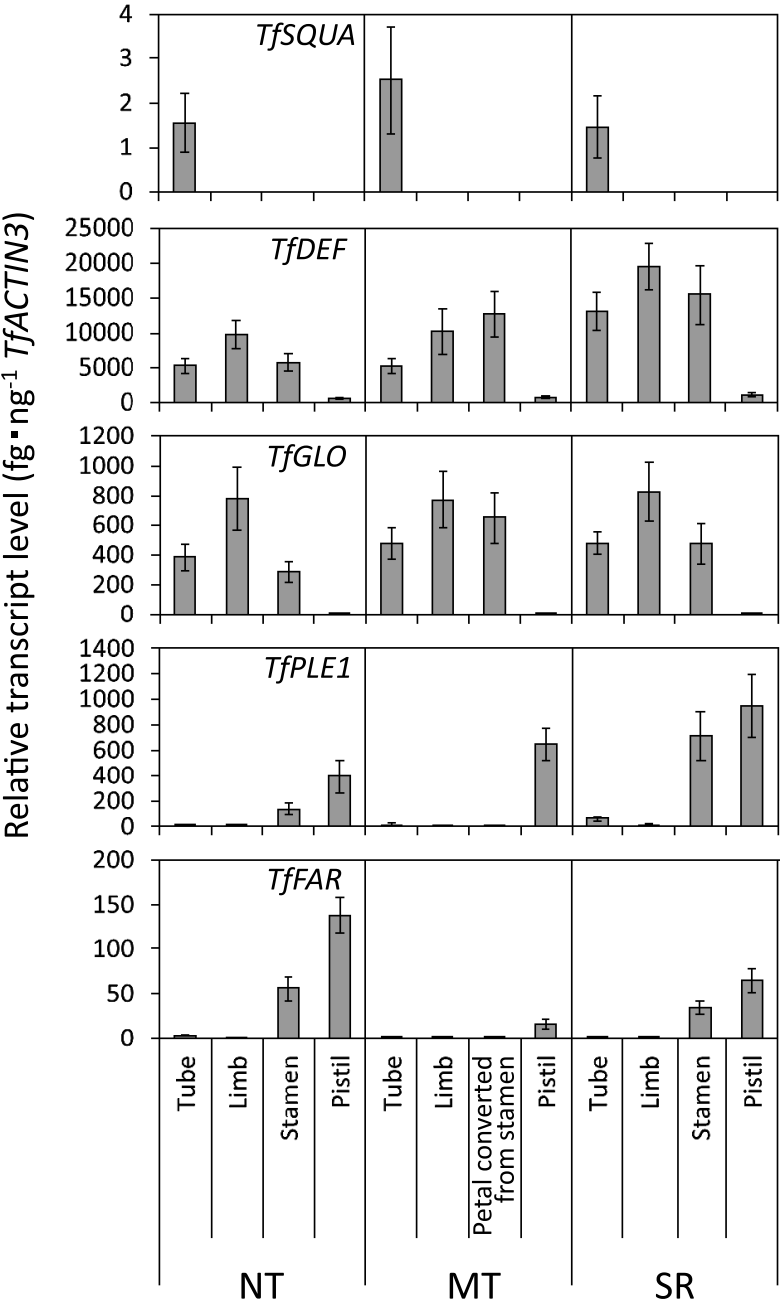

Fig. 5. Expression of floral homeotic genes in young flower buds of the "Petaloid" mutant. Flower buds in the late petal development stage (late stage $6,14-16 \mathrm{~mm}$ corolla length) were used for the experiment. NT, normal type; MT, mutant type; and SR, flower with complete somatic reversion. Vertical bars indicate SE $(n=3)$.

sion of the pistil to new flowers, as observed in mild phenotypes of agamous (ag) of Arabidopsis thaliana (Yanofsky et al., 1990) and plena (ple) of A. majus (Carpenter and Coen, 1990).

Identification of the mutation responsible for the "Petaloid" phenotype

When the genomic sequence, including the fulllength open reading frames of the $\mathrm{C}$-class floral homeotic genes, was amplified by PCR, 4.0- and $4.8-\mathrm{kbp}$ DNA fragments of TfPLE1 and TfFAR, respectively, were detected in all of the NT, MT, and SR flowers, whereas an additional 7.7-kbp fragment of TfFAR was detected in the MT flower (Fig. 6). These results suggest the insertion of the 2.9-kbp element Ttfl in TfFAR in MT flowers. Thus, we cloned the $4.8-$ and $7.7-\mathrm{kbp}$ fragments of TfFAR. Sequence analysis of the $4.8-\mathrm{kbp}$ fragment showed that TfFAR consists of 9 exons and 8 
introns (Fig. 7A, LC075590). Further, sequence analysis of the 7.7-kbp fragment showed insertion of Ttfl (2893 bp) in the long 2nd intron (2401 bp) of TfFAR with the left terminal placed in the $5^{\prime}$ direction (Fig. 7A). The long 2nd intron is also known from the $A G$ of A. thaliana and PLE of A. majus, which belong to $\mathrm{C}$-class floral homeotic genes. There is evidence that these introns contain regulatory elements, which contribute to the control of spatial and temporal expression (Bradley et al., 1993; Busch et al., 1999; Deyholos and Sieburth, 2000; Hong et al, 2003; Sieburth and Meyerowitz, 1997). Insertion of a DNA transposon, Tam3, into the 2nd intron of PLE inhibits expression of, or induces ectopic expression of, PLE depending on the site and direction of Tam 3 insertion (Bradley et al., 1993). Inhibition of PLE expression by Tam 3 induces the conversion of stamens and carpels into petals and new flowers, respectively. Therefore, Ttfl insertion into the 2nd intron of TfFAR in torenia inhibited TfFAR expression, which induced the conversion of stamens into petals.

It should be noted that Ttf1 insertion inhibited TfFAR expression to a greater extent in stamens than in pistils in early stage 6 (Fig. 4). Thus, Ttf1 insertion may have

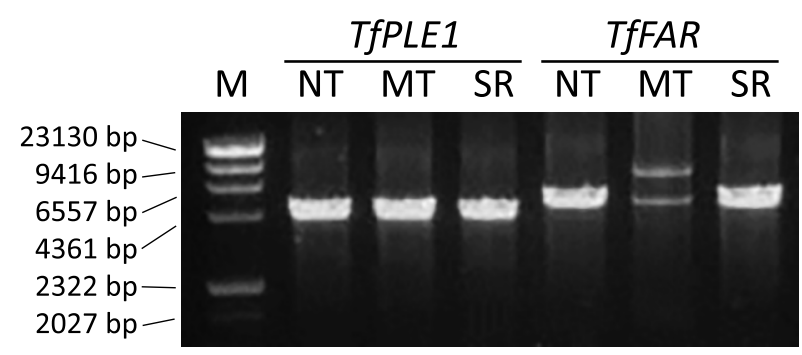

Fig. 6. PCR products from genomic DNA, including full-length class-C floral homeotic genes. NT, normal type; MT, mutant type; and SR, flower with complete somatic reversion. M, molecular weight marker. affected organ-specific regulation of TfFAR expression. A regulatory element $\mathrm{CArG}$ box, which inhibits ectopic expression of C-class floral homeotic genes (Hong et al., 2003), was located 24-bp 5'-upstream of the Ttf1 insertion site of TfFAR. However, this structure does not appear to inhibit TfFAR expression through reduction of the CArG box function, which would induce ectopic expression of TfFAR (Hong et al., 2003). We could not find, near the Ttfl insertion site, any other known regulatory sequences involved in spatial and temporal regulation of C-class floral homeotic genes including the LEAFY and WUSCHEL binding sites (Busch et al., 1999; Lohmann et al., 2001), AAGAAT box and CCAAT box (Hong et al., 2003).

The 3-bp target duplication sequences flanking Ttf1 (Fig. 7B, TTT) were not present in NT flowers, which were formed on the integration of Ttfl, as observed in other En/Spm transposons (Gierl and Saedler, 1992). Since the target duplication sequence is TGA when Ttf1 is integrated in TfMYB1 (Nishijima et al., 2013), the target sequence for Ttfl integration is not only TGA, but also TTT. In SR flowers, the total target duplication sequence, TTTTTT, was converted to the new 3- and 4-bp sequences TTT and TTTT (Fig. 7B), which were generated as footprints of $T t f 1$ excision.

\section{Correspondence of the TfFAR genotype with flower traits}

To confirm whether the TfFAR genotype actually corresponds with the observed floral traits, $\mathrm{S}_{1}$ segregants for the "Petaloid" trait were assessed to see whether they contained TfFAR with or without Ttfl insertion, i.e. alleles $f^{7 t f l}$ or $F$, respectively, using qPCR. There was clear correspondence between the TfFAR genotype and the floral traits (Fig. 8). Most MT plants exhibited a high quantity of $f^{\text {It } f l}$, although a small quantity of $F$ was detected. This result indicates that the genotype of MT plants is essentially $f^{7 t f l} / f^{7 t f l}$, although a small amount of

A

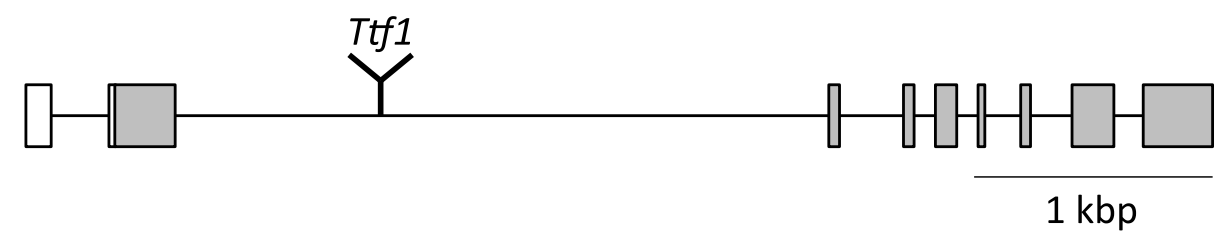

B

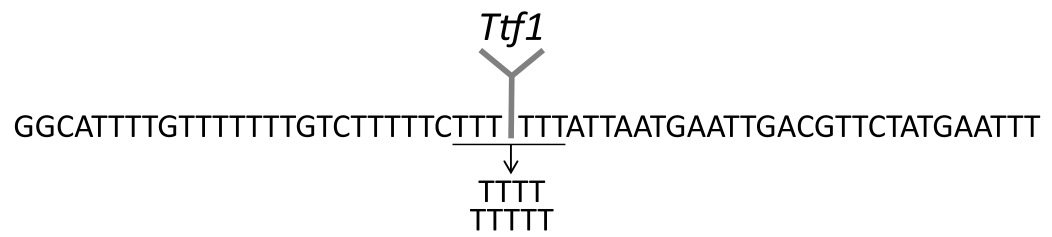

Fig. 7. Insertion of Ttfl in TfFAR. A: Structure of TfFAR carrying Ttfl. Open square, 5' UTR; grey square, coding region; horizontal line, intron. B: DNA sequence around Ttfl insertion site of TfFAR and footprint sequences formed by excision of Ttf1 (TTTT and TTTTT). Sequences of TTT underlined in the TfMYB1 sequence are 3-bp target duplications. 


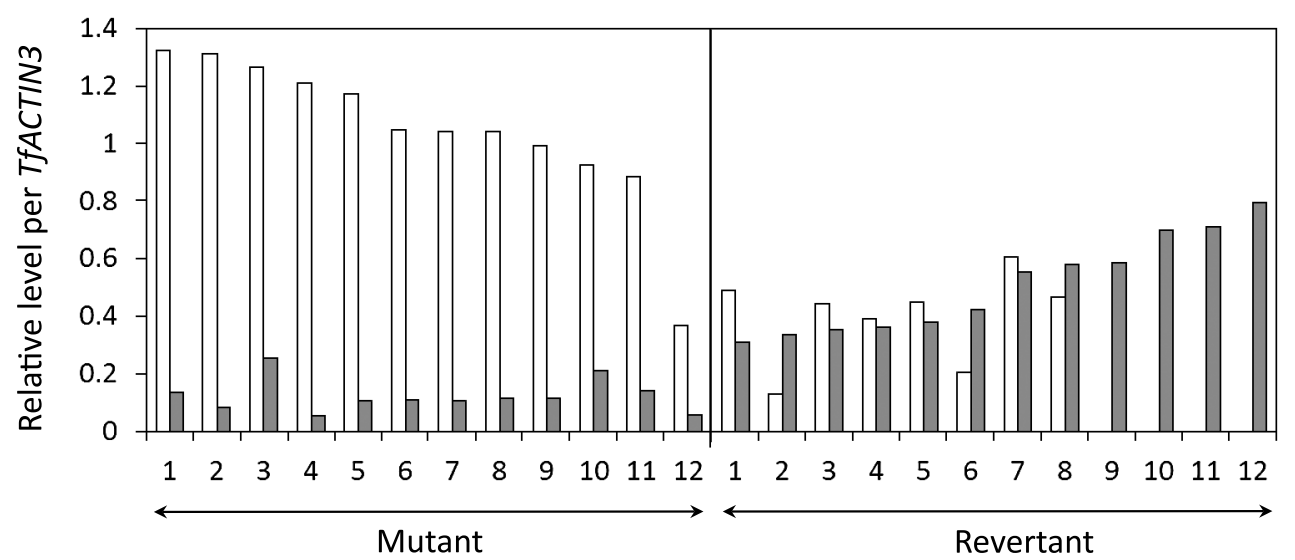

Fig. 8. TfFAR genotypes analysed by $\mathrm{qPCR}$ in $\mathrm{S}_{1}$ plants derived from somatic revertant flowers. The open column indicates a mutant-type allele with $T t f 1$ insertion $\left(f^{7 t f 1}\right)$, while the grey column indicates a normal-type allele without $T t f 1$ insertion $(F)$. For clarity, data were arranged in descending order in quantities of $f^{I t f l}$ of mutant type plants and ascending order in quantities of $F$ of revertant plants.

somatic revertant tissue is included. In contrast, all GR plants exhibited a high quantity of $F$. $f^{I t f l}$ was detected in 8 out of 12 GR plants, suggesting they have the $F / f^{\text {It } f I}$ genotype, whereas the other 4 GR plants had no detectable $f^{T f f}$, suggesting they have the $F / F$ genotype. Overall, we concluded that the "Petaloid" mutation is

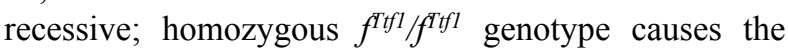
"Petaloid" phenotype, whereas the $F / f^{\text {Itf } I}$ and $F / F$ genotypes cause the normal phenotype.

\section{Inactivation of Ttf1 transposition}

Since Ttfl is a non-autonomous element, the genetic separation of Ttfl from the unidentified factor activating Ttfl transposition may stabilize Ttfl-caused mutations, as shown in rice cotransfected with transposon $D s$ and the transposase gene $A c$ of maize (Shimamoto et al., 1993). Therefore, we crossed the "Petaloid" mutant and 'Common Violet' in which the Ttfl transposition is inactive. All $48 \mathrm{~F}_{1}$ plants bore single flowers (Table 2). In $F_{2}$ plants descended from self-pollination of $3 \mathrm{~F}_{1}$ plants, 6 of 60 plants $(10 \%)$ bore double flowers and 54 of 60 plants $(90 \%)$ bore only single flowers. Within the first 40 flowers opened per plant, the 4 unstable plants bore $18-30(36 \%-75 \%)$ revertant flowers, while 2 moderately stable plants bore 2 and $4(5 \%$ and $10 \%$, respectively) revertant flowers. qPCR analysis of genomic DNA of those double-flowered plants showed that 2 moderately stable double-flowered plants had the complete $f^{I t f l} / f^{I t f l}$ genotype with no reversion to $F$ (Fig. 9), while the 4 unstable plants showed substantial $F$ allele levels in mutant flowers analysed. Therefore, phenotypic reversion observed in the 2 moderately stable plants is not likely due to genetic reversion but to inconsistency of the double-flowered phenotype expressed by the $\mathrm{far}^{T t f l} / \mathrm{far}^{T t f l}$ genotype.

These results suggest that the mutability of the double-flowered trait was reduced through the separation of the Ttfl insertion and the unidentified activator of Ttfl transposition. The "Petaloid" mutation is reces-

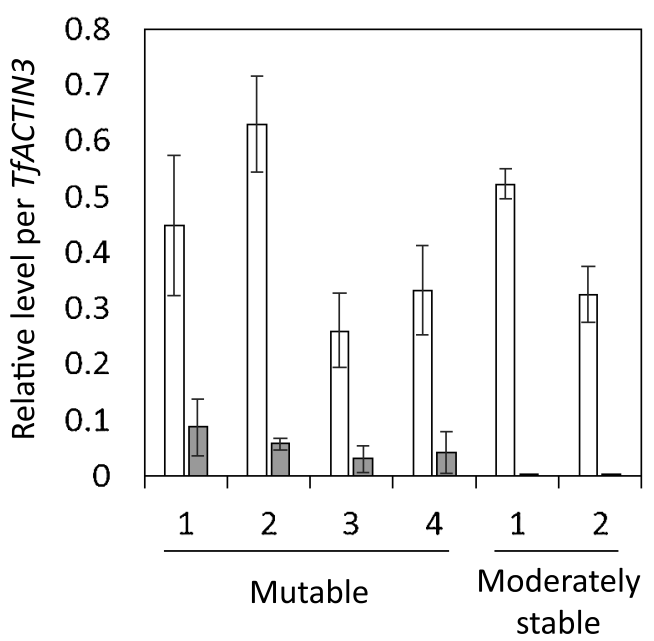

Fig. 9. Quantification of TfFAR genotypes in mutable and moderately stable double-flowered $\mathrm{F}_{2}$ plants derived from a cross between "Petaloid" mutant and normal type plant. The open column indicates a mutant-type allele with $T t f 1$ insertion $\left(f^{T t f I}\right)$, while the grey column indicates a normal-type allele without Ttfl insertion $(F)$. Numbers in horizontal axis indicate plant number. Vertical bars indicate SE $(\mathrm{n}=3)$.

sive as discussed above, while trait of active Ttfl transposition inherits dominantly. Thus, $19 \%$ mutable and $19 \%$ stable double-flowered plants should have theoretically occurred in the $\mathrm{F}_{2}$ generation; however, only $7 \%$ mutable and $3 \%$ stable double-flowered plants occurred in our experiment. This result suggests that $T t f 1$ was excised from TfFAR during growth and reproduction.

\section{Function of torenia C-class floral homeotic genes}

In angiosperms, a duplication event in the $A G$ family resulted in the $e u A G$ and PLE lineages within the core eudicots (Kramer et al., 2004). Genes in the $e u A G$ and $P L E$ lineages show species-dependent functional differences. Genes belonging to the $e u A G$ lineage, such as $A G$ of A. thaliana (Favaro et al., 2003; Yanofsky et al., 1990), TOMATO AGAMOUS1 of the tomato (Pnueli 
et al., 1994), PMADS3 of the petunia (Kapoor et al., 2002, 2005; Kater et al., 1998; Tsuchimoto et al., 1993), and DUPLICATED of the Japanese morning glory (Nitasaka, 2003) are necessary for stamen and carpel development. In contrast, genes belonging to the PLE lineage, such as SHATTERPROOF1/2 of A. thaliana, are necessary for dehiscence zone formation in the silique (Liljegren et al., 2000; Pinyopich et al., 2003). TOMATO AGAMOUS-likel of the tomato is necessary for fruit development and maturation (Itkin et al., 2009; Vrebalov et al., 2009). The functions of FBP6 of petunia (Kater et al., 1998) and PEONY of Japanese morning glory (Nitasaka, 2003) are unknown.

However, $A G$-family genes in $A$. majus, which like torenia belongs to the Lamiales, have the opposite functional assignment from those in the aforementioned 4 plant species; the PLE-lineage gene PLE is necessary for the development of stamens and carpels (Bradley et al., 1993; Carpenter and Coen, 1990), whereas the euAG lineage gene FAR is necessary for pollen development (Davies et al., 1999). FAR also has weak and partial function for the promotion of stamen and carpel development because a double mutation of PLE and FAR enhances homeotic conversion of carpels into petals and indeterminate development of flowers more extensively than mutation of PLE alone (Bradley et al., 1993). In summary, either $e u A G$ - or PLE-lineage genes function primarily as C-class floral homeotic genes, and genes of the other lineage either perform a supplementary C-class floral homeotic function, or have a different function.

In the present study, stamens of torenia were converted to petals through the inhibition of TfFAR expression caused by Ttfl insertion. This result suggests that TfFAR functions mainly for determination of stamen identity. This is contrary to the understanding that the PLE lineage functions mainly in the determination of stamen and carpel identity in A.majus, as mentioned above. Therefore, it is evident that this divergence in functional assignment of $A G$ - and PLE-lineage genes occurred after the phylogenic differentiation of Lamiales.

Although the function of TfPLE1 has not yet been identified, inconsistency of double-flowerd traits shown in the plant with stabilized $f_{a r}{ }^{T t f l} / f a r^{T t f l}$ genotype may indicate that reduction of TfFAR function is not sufficient for complete conversion of stamens to petals; i.e., TfPLE functions partially for determination of stamen identity with TfFAR. In addition, it should be noted that TfPLE1 expression decreased in petals converted from stamens (Figs. 4 and 5). In A. majus, homeotic conversion of floral organs into staminoid organs through ectopic expression of PLE induces expression of FAR, which is necessary for pollen development (Davies et al., 1999). In Arabidopsis, $A G$ induces $S H P 1 / 2$ which are necessary for dehiscence zone formation in the silique as mentioned above (Gómez-Mena et al., 2004).
These findings indicate that either $e u A G$ or PLE lineage genes functioning as $\mathrm{C}$-class floral homeotic genes induce the other lineage genes necessary for stamen and carpel development. Therefore, if TfPLE1 performs a function necessary for stamen development, it follows that TfPLE1 expression was reduced in petals converted from stamens (Fig. 4). Those 2 hypothesis for possible TfPLE function remain to be elucidated.

\section{Future perspectives}

Construction of transgenic plants overexpressing the chimeric $A G$ repressor was previously attempted to induce double flowers in torenia, but resulted in only partial reduction of C-class function and incomplete conversion of stamens into petals (Narumi et al., 2008). The "Petaloid" mutant reported in the present paper is the first published torenia germplasm bearing double flowers with complete conversion of stamens into petals. Further, genetic separation between the Ttfl insertion responsible for the desired phenotype, and the unidentified factor activating Ttf1 transposition was found to be effective to increase stability of the mutant trait.

Although the double-flowered mutation is currently caused solely by conversion of stamens into petals, it may be possible to further increase the ornamental value of double-flowered torenia by inducing the conversion of carpels into new indeterminate flowers. This result could be attained in the following 2 ways. One is to induce mutations that more strongly inhibit TfFAR function. In the Japanese morning glory, the reduction of C-class function by insertion of the transposon $T p n$ in the C-class gene DUPLICATED (DP) induces conversion of stamens into petals (Nitasaka, 2003, 2007). Furthermore, deletion of a large portion of $D P$, presumably as a result of incomplete excision of Tpn and subsequent illegitimate recombination of $D P$, induces formation of an indeterminate double flower. In the present study, carpels of torenia remained unaltered even when TfFAR expression was inhibited by Ttf1 insertion, probably because TfFAR expression remained in the pistil, as discussed above. Thus, the null mutation of TfFAR may substitute indeterminately developed new flowers for the pistil. The other way is to obtain double mutants of TfFAR and TfPLE1 to inhibit C-class floral homeotic function more intensively. This will be effective if TfPLE1 has partial C-class function, similar to FAR of A.majus, as mentioned above (Davies et al., 1999).

\section{Acknowledgements}

We thank Mrs. Tomoko Kurobe, Mrs. Toshie Iida, and Mrs. Setsuko Kimura for their technical assistance.

\section{Literature Cited}

Aida, R., S. Kishimoto, Y. Tanaka and M. Shibata. 2000. Modification of flower color in torenia (Torenia fournieri Lind.) by 
genetic transformation. Plant Sci. 153: 33-42.

Bowman, J. L., D. R. Smyth and E. M. Meyerowitz. 1991. Genetic interactions among floral homeotic genes of Arabidopsis. Development 112: 1-20.

Bradley, D., R. Carpenter, H. Sommer, N. Hartley and E. Coen. 1993. Complementary floral homeotic phenotypes result from opposite orientations of a transposon at the plena locus of Antirrhinum. Cell 72: 85-95.

Busch, M. A., K. Bomblies and D. Weigel. 1999. Activation of a floral homeotic gene in Arabidopsis. Science 285: 585-587.

Carpenter, R. and E. S. Coen. 1990. Floral homeotic mutations produced by transposon-mutagenesis in Antirrhinum majus. Genes Dev. 4: 1483-1793.

Coen, E. S. and E. M. Meyerowitz. 1991. The war of the whorls: genetic interactions controlling flower development. Nature 353: 31-37.

Davies, B., P. Motte, E. Keck, H. Saedler, H. Sommer and Z. Schwarz-Sommer. 1999. PLENA and FARINELLI: redundancy and regulatory interactions between two Antirrhinum MADS-box factors controlling flower development. EMBO J. 18: 4023-4034.

Deyholos, M. K. and L. E. Sieburth. 2000. Separable whorlspecific expression and negative regulation by enhancer elements within the AGAMOUS second intron. Plant Cell 12: 1799-1810.

Drews, G. N., J. L. Bowman and E. M. Meyerowitz. 1991. Negative regulation of the Arabidopsis homeotic gene AGAMOUS by the APETALA2 product. Cell 65: 991-1002.

Favaro, R., A. Pinyopich, R. Battaglia, M. Kooiker, L. Borghi, G. Ditta, M. F. Yanofsky, M. M. Kater and L. Columbo. 2003. MADS-box protein complexies control carpel and ovule development in Arabidopsis. Plant Cell 15: 2603-2611.

Gierl, A. and H. Saedler. 1992. Plant-transposable elements and gene tagging. Plant Mol. Biol. 19: 39-49.

Gómez-Mena, C., S. de Folter, M. M. R. Costa, G. C. Angenent and R. Sablowski. 2004. Transcriptional program controlled by the floral homeotic gene AGAMOUS during early organogenesis. Development 132: 429-438.

Goodrich, J., R. Carpenter and E. S. Coen. 1992. A common gene regulates pigmentation pattern in diverse plant species. Cell 68: 955-964.

Gustafson-Brown, C., B. Savidge and M. F. Yanofsky. 1994. Regulation of the Arabidopsis floral homeotic gene APETALA1. Cell 76: 131-143.

Hong, R. L., L. Hamaguchi, M. A. Busch and D. Weigel. 2003. Regulatory elements of the floral homeotic gene AGAMOUS identified by phylogenic footprinting and shadowing. Plant Cell 15: 1296-1309.

Inagaki, Y., Y. Hisatomi, T. Suzuki, K. Kasahara and S. Iida. 1994. Isolation of a Suppressor-Mutator/Enhancer-like transposable element, Tpn1, from Japanese morning glory bearing variegated flowers. Plant Cell 6: 375-383.

Itkin, M., H. Seybold, D. Breitel, I. Rogachev, S. Meir and A. Aharoni. 2009. The TOMATO AGAMOUS-LIKE 1 is a component of the fruit ripening regulatory network. Plant J. 60: 1081-1095.

Kapoor, M., A. Baba, K. Kubo, K. Shibuya, K. Matsui, Y. Tanaka and H. Takatsuji. 2005. Transgene-triggered, epigenetically regulated ectopic expression of a flower homeotic gene pMADS3 in petunia. Plant J. 43: 649-661.

Kapoor, M., S. Tsuda, Y. Tanaka, T. Mayama, Y. Okuyama, S. Tsuchimoto and H. Takatsuji. 2002. Role of petunia pMADS3 in determination of floral organ and meristem identity, as revealed by its loss of function. Plant J. 32: 115127.
Kater, M. M., L. Colombo, J. Franken, M. Busscher, S. Masiero, M. M. Van Lookeren and G. C. Angenent. 1998. Multiple AGAMOUS homologs from cucumber and petunia differ in their ability to induce reproductive organ fate. Plant Cell 10: 171-182.

Kramer, E. M., M. A. Jaramillo and V. S. Di Stilio. 2004. Patterns of gene duplication and functional evolution during the diversification of the AGAMOUS subfamily of MADS box genes in angiosperms. Genetics 166: 1011-1023.

Lenhard, M., A. Bohnert, G. Jurgens and T. Laux. 2001. Termination of stem cell maintenance in Arabidopsis floral meristems by interactions between WUSCHEL and AGAMOUS. Cell 105: 805-814.

Liljegren, S. J., G. S. Ditta, Y. Eshed, B. Savidge, J. L. Bowmann and M. F. Yanofsky. 2000. SHATERPROOF MADS-box genes control seed dispersal in Arabidopsis. Nature 404: 766-770.

Lohmann, J. U., R. L. Hong, M. Hobe, M. A. Busch, F. Parcy, R. Simon and D. Weigel. 2001. A molecular link between stem cell regulation and floral patterning in Arabidopsis. Cell 105: 793-803.

Matsubara, K., H. Kodama, H. Kokubun, H. Watanabe and T. Ando. 2005. Two novel transposable elements in a cytochrome P450 gene govern anthocyanin biosynthesis of commercial petunias. Gene 358: 121-126.

Miyazaki, K., C. Kuwayama, K. Kageyama, S. Matsumoto and H. Fukui. 2007. Relation between interspecific compatibility and phylogenic analysis in Torenia. Shokubutsu Kankyo Kogaku 19: 66-73 (In Japanese with English abstract).

Morise, N. 2001. Torenia. p. 567-568. In: Nosangyosonbunkakyokai (ed.). Nogyogijutsutaikei (In Japanese). Nosangyosonbunkakyokai, Tokyo.

Nakajima, T., K. Matsubara, H. Kodama, H. Kokubun, H. Watanabe and T. Ando. 2005. Insertion and excision of a transposable element governs the red floral phenotype in commercial petunias. Theor. Appl. Genet. 110: 1038-1043.

Narumi, T., R. Aida, T. Niki, T. Nishijima, N. Mitsuda, K. Hiratsu, M. Ohme-Takagi and N. Ohtsubo. 2008. Chimeric AGAMOUS repressor induces serrated petal phenotype in Torenia fournieri similar to that induced by cytokinin application. Plant Biotechnol. 25: 45-54.

National Agriculture and Food Research Organaization. 2006. Torenia. p. 1112. In: National Agriculture and Food Research Organization (ed.). NAROPEDIA (In Japanese). Nosangyosonbunkakyokai, Tokyo.

Niki, T., M. Hirai, T. Niki, A. Kanno and T. Nishijima. 2012. Role of homeotic genes in the morphology of forchlorfenuron-induced paracorollas in Torenia fournieri Lind. J. Japan. Soc. Hort. Sci. 81: 204-212.

Nishijima, T. 2012. Large flower size: molecular basis and role of cytokinin. J. Japan. Soc. Hort. Sci. 81: 129-139.

Nishijima, T. and K. Shima. 2006. Change in flower morphology of Torenia fournieri Lind. induced by forchlorfenuron application. Sci. Hortic. 109: 254-261.

Nishijima, T., Y. Morita, K. Sasaki, M. Nakayama, H. Yamaguchi, N. Otsubo, T. Niki and T. Niki. 2013. A torenia (Torenia fournieri Lind. ex Fourn.) novel mutant 'Flecked' produces variegated flowers by insertion of a DNA transposon into a $R 2 R 3-M Y B$ gene. J. Japan. Soc. Hort. Sci. 82: $39-50$.

Nitasaka, E. 2003. Insertion of an En/Spm-related transposable element into a floral homeotic gene DUPLICATED causes a double flower phenotype in the Japanese morning glory. Plant J. 36: 522-531.

Nitasaka, E. 2007. Morphological mutants induced by transposa- 
ble elements in the Japanese morning glory. Gamma Field Symp. 46: 73-78.

Oshima, M., S. Hirose, M. Kawagishi, K. Kawaguchi, F. Abe, M. Fujisato and K. Nishizawa. 2010. Current status of development of transgenic crops in Japan and forthcoming challenges. Tokugikon 556: 7-19 (In Japanese).

Pinyopich, A., G. S. Ditta, B. Savidge, S. J. Liljegren, E. Baumann, E. Wisman and M. F. Yanofsky. 2003. Assessing the redundancy of MADS-box genes during carpel and ovule development. Nature 424: 85-88.

Pnueli, L., D. Hareven, S. D. Rounsley, M. F. Yanofsky and E. Lifschitz. 1994. Isolation of the tomato AGAMOUS gene $T A G 1$ and analysis of its homeotic role in transgenic plants. Plant Cell 6: 163-173.

Sasaki, K., R. Aida, T. Niki, H. Yamaguchi, T. Narumi, T. Nishijima, Y. Hayashi, H. Ryuto, N. Fukunishi, T. Abe and N. Otsubo. 2008. High-efficiency improvement of transgenic torenia flowers by ion beam irradiation. Plant Biotechnol. 25: 81-90.

Sasaki, K., H. Yamaguchi, M. Nakayama, R. Aida and N. Otsubo. 2014. Co-modification of class B genes TfDEF and TfGLO in Torenia fournieri Lind. alters both flower morphology and inflorescence architecture. Plant Mol. Biol. 86: 319-334.

Shimamoto, K., C. Miyazaki, H. Hashimoto, T. Izawa, K. Itoh, R.
Terada, Y. Inagaki and S. Iida. 1993. Trans-activation and stable integration of the maize transposable element $D s$ cotransfected with the $A c$ transposase gene in transgenic rice plant. Mol. Gen. Genet. 239: 354-360.

Sieburth, L. E. and E. M. Meyerowitz. 1997. Molecular dissection of the AGAMOUS control region shows that cis elements for spatial regulation are located intragenically. Plant Cell 9: 355-365.

Tsuchimoto, S., A. R. van der Krol and N.-H. Chua. 1993. Ectopic expression of PMADS3 in transgenic petunia phenocopies the petunia blind mutant. Plant Cell 5: 843-853.

Yamaguchi, H., T. Niki, T. Niki and T. Nishijima. 2010. Morphological property and role of homeotic genes in paracorolla development of Antirrhinum majus. J. Japan. Soc. Hort. Sci. 79: 192-199.

Yanofsky, M. F., H. Ma, J. L. Bowman, G. N. Drews, K. A. Feldman and E. M. Meyerowitz. 1990. The protein encoded by the Arabidopsis homeotic gene agamous resembles transcription factors. Nature 346: 35-39.

Vrebalov, J., I. L. Pan, A. J. Arroyo, R. McQuinn, M. Chung, M. Poole, J. Rose, G. Seymour, S. Grandillo, J. Giovannoni and V. F. Irish. 2009. Fleshy fruit expansion and ripening are regulated by the tomato SHATTERPROOF gene TAGL1. Plant Cell 21: 3041-3062. 